\title{
Highly Enantioselective Synthesis of Chiral 3-Substituted Indolines by Catalytic Asymmetric Hydrogenation of Indoles
}

Ryoichi Kuwano, ${ }^{\dagger}, *$ Kohei Kaneda, ${ }^{\dagger}$ Takashi Ito, ${ }^{\ddagger}$ Koji Sato, ${ }^{\ddagger}$ Takashi Kurokawa, ${ }^{\ddagger}$ and Yoshihiko Ito ${ }^{\ddagger * *}$

$\dagger$ Department of Chemistry, Graduate School of Sciences, Kyushu University, 6-10-1 Hakozaki, Higashi-ku, Fukuoka 8128581, Japan

\# Department of Synthetic Chemistry \& Biological Chemistry, Graduate School of Engineering, Kyoto University, Sakyoku, Kyoto 606-8501, Japan

\section{Supporting Information}

General and Materials. Specific rotations were measured with a JASCO P-1020 polarimeter. NMR spectra were measured with Varian GEMINI-2000 (7.0 T magnet) or Bruker AVANCE 400 (9.4 $\mathrm{T}$ magnet) spectrometer. Flash column chromatograhies were performed with silica gel 60 (230-400 mesh, Merck).

All reactions were conducted under nitrogen atomosphere. 2-Propanol and DMF were distilled from $\mathrm{CaH}_{2}$ under nitrogen atomosphere. $\mathrm{CH}_{2} \mathrm{Cl}_{2}$ was distilled from $\mathrm{P}_{2} \mathrm{O}_{5}$. THF and toluene were distilled from sodium benzophenone ketyl. [Rh(nbd) $\left.)_{2}\right] \mathrm{SbF}_{6},{ }^{1}(S, S)-(R, R)-$ and $(R, R)-(S, S)-\mathrm{PhTRAP}^{2}{ }^{2} \mathbf{5}^{3}$ and $7^{4}$ were prepared according to literatures. Sodium bis(2-methoxyethoxy)aluminum dihydride was purchased from TCI.

General procedure of the catalytic asymmetric hydrogenation of 3-Substituted Indoles: A mixture of $\left[\mathrm{Rh}(\mathrm{nbd})_{2}\right] \mathrm{SbF}_{6}(2.6 \mathrm{mg}, 5.0 \mu \mathrm{mol})$ and $(S, S)-(R, R)$-PhTRAP $(4.0 \mathrm{mg}, 5.0 \mu \mathrm{mol})$ in 2propanol $(2.0 \mathrm{ml})$ was stirred vigorously at room temperature for $10 \mathrm{~min}$. The mixture was transferred by a cannula to a nitrogen-filled stainless steel autoclave, in which 3 -substituted indole $\mathbf{1}(0.50 \mathrm{mmol})$ and $\mathrm{Cs}_{2} \mathrm{CO}_{3}(16.2 \mathrm{mg}, 50 \mu \mathrm{mol})$ were placed beforehand. Hydrogen was introduced into the reaction vessel until the pressure gauge indicated $50 \mathrm{~kg} / \mathrm{cm}^{2}$. The reaction mixture was stirred at $80{ }^{\circ} \mathrm{C}$ for $24 \mathrm{~h}$. After the solvent was evaporated, the residue was purified by flash column chromatography on silica gel (hexanes/EtOAc) to obtain the desired 3-substituted indoline 2.

(S)-3-Methyl-1-(p-toluenesulfonyl)indoline (2c) (Table 1, Entry 4). The general procedure was followed with 3-methyl-1-( $p$-toluenesulfonyl)indole (1c) $(143 \mathrm{mg}, 0.50 \mathrm{mmol})$ to give $(S)$-2c (138 $\mathrm{mg}, 96 \%$ ). The enantiomeric excess of the product was determined to be $98 \%$ ee by HPLC analysis with a chiral stationary phase column, CHIRALCEL OD-H $(4.6 \mathrm{~mm} \phi \times 25 \mathrm{~cm}, 4 \%$ 2-propanol in hexane): colorless solid; $[\alpha]^{20}{ }_{\mathrm{D}}=+29.4\left(c 1.06, \mathrm{CHCl}_{3}\right) ;{ }^{1} \mathrm{H} \mathrm{NMR}\left(300 \mathrm{MHz}, \mathrm{CDCl}_{3}, \mathrm{TMS}\right) \delta 1.11(\mathrm{~d}, J$ $=7.0 \mathrm{~Hz}, 3 \mathrm{H}), 2.36(\mathrm{~s}, 3 \mathrm{H}), 3.19$ (double quintet, $J=8.6,7.0 \mathrm{~Hz}, 1 \mathrm{H}), 3.42(\mathrm{dd}, J=7.0,10.4 \mathrm{~Hz}, 1 \mathrm{H})$, $4.08(\mathrm{dd}, J=8.6,10.4 \mathrm{~Hz}, 1 \mathrm{H}), 6.96-7.08(\mathrm{~m}, 2 \mathrm{H}), 7.16-7.26(\mathrm{~m}, 3 \mathrm{H}), 7.64(\mathrm{~d}, J=8.4 \mathrm{~Hz}, 1 \mathrm{H}), 7.68$ $(\mathrm{d}, J=8.4 \mathrm{~Hz}, 2 \mathrm{H}) ;{ }^{13} \mathrm{C}\left\{{ }^{1} \mathrm{H}\right\}$ NMR $\left(75 \mathrm{MHz}, \mathrm{CDCl}_{3}\right) \delta 19.4,21.4,34.6,57.5,114.9,123.8,124.0$, 127.4, 127.9, 129.7, 134.1, 136.9, 141.6, 144.1; Anal. Calcd for $\mathrm{C}_{16} \mathrm{H}_{17} \mathrm{NO}_{2} \mathrm{~S}: \mathrm{C}, 66.87 ; \mathrm{H}, 5.96$; N, 4.87. Found: C, 66.84; H, 5.96; N, 4.73.

$(\boldsymbol{R})-3-M e t h y l-1-(p$-toluenesulfonyl)indoline (2c). The general procedure was followed with 3methyl-1-( $p$-toluenesulfonyl)indole (1c) $(285 \mathrm{mg}, 1.00 \mathrm{mmol})$ and $(R, R)-(S, S)$-PhTRAP to give $(R)$-2c $(280 \mathrm{mg}, 98 \%)$. The enantiomeric excess of the product was determined to be $98 \%$ ee by HPLC analysis with a chiral stationary phase column, CHIRALCEL OD-H $(4.6 \mathrm{~mm} \phi \times 25 \mathrm{~cm}, 4 \% 2-$ propanol in hexane): colorless solid; $[\alpha]_{\mathrm{D}}^{25}=-32.4\left(c 1.54, \mathrm{CHCl}_{3}\right)$.

(+)-3-(2-Propyl)-1-(p-toluenesulfonyl)indoline (2f) (Table 2, Entry 1). The general procedure except amount of solvent $(1 \mathrm{ml})$ and reaction time $(48 \mathrm{~h})$ was followed with 3-(2-propyl)-1-( $p$ toluenesulfonyl)indole (1f) $(157 \mathrm{mg}, 0.50 \mathrm{mmol})$ to give $\mathbf{2 f}(152 \mathrm{mg}, 94 \%)$. The enantiomeric excess of the product was determined to be $97 \%$ ee by HPLC analysis with a chiral stationary phase column, 
CHIRALCEL OD-H (4.6 mm $\phi \times 25 \mathrm{~cm}, 20 \%$ 2-propanol in hexane): colorless oil; $[\alpha]^{20}=-19.1(c$ 1.06, $\left.\mathrm{CHCl}_{3}\right) ;{ }^{1} \mathrm{H} \mathrm{NMR}\left(400 \mathrm{MHz}, \mathrm{CDCl}_{3}, \mathrm{TMS}\right) \delta 0.63(\mathrm{~d}, J=6.8 \mathrm{~Hz}, 3 \mathrm{H}), 0.88(\mathrm{~d}, J=6.8 \mathrm{~Hz}, 3 \mathrm{H})$, 1.87 (double septet, $J=4.8,6.8 \mathrm{~Hz}, 1 \mathrm{H}), 2.37(\mathrm{~s}, 3 \mathrm{H}), 3.11(\mathrm{dt}, J=10.5,5.2 \mathrm{~Hz}, 1 \mathrm{H}), 3.73(\mathrm{dd}, J=5.5$, $10.5 \mathrm{~Hz}, 1 \mathrm{H}), 3.81(\mathrm{dd}, J=10.0,10.5 \mathrm{~Hz}, 1 \mathrm{H}), 6.96(\mathrm{t}, J=7.5 \mathrm{~Hz}, 1 \mathrm{H}), 7.06(\mathrm{~d}, J=7.5 \mathrm{~Hz}, 1 \mathrm{H}), 7.19$ $(\mathrm{t}, J=8.0 \mathrm{~Hz}, 1 \mathrm{H}), 7.23(\mathrm{~d}, J=8.0 \mathrm{~Hz}, 2 \mathrm{H}), 7.64(\mathrm{~d}, J=8.0 \mathrm{~Hz}, 1 \mathrm{H}), 7.71(\mathrm{~d}, J=8.0 \mathrm{~Hz}, 2 \mathrm{H}) ;{ }^{13} \mathrm{C}\left\{{ }^{1} \mathrm{H}\right\}$ NMR $\left(100.5 \mathrm{MHz}, \mathrm{CDCl}_{3}\right) \delta$ 17.3, 20.0, 21.5, 31.3, 45.8, 51.7, 114.1, 123.2, 125.0, 127.3, 127.9, 129.6, 133.9 (2C), 142.2, 144.0; Anal. Calcd for $\mathrm{C}_{18} \mathrm{H}_{21} \mathrm{NO}_{2} \mathrm{~S}: \mathrm{C}, 68.54 ; \mathrm{H}, 6.71 ; \mathrm{N}, 4.44$. Found: C, 68.47; H, 6.70; N, 4.39.

(+)-3-Phenyl-1-(p-toluenesulfonyl)indoline (2g) (Table 2, Entry 2). The general procedure was followed with 3-phenyl-1-(p-toluenesulfonyl)indole (1c) $(174 \mathrm{mg}, 0.50 \mathrm{mmol}),\left[\mathrm{Rh}(\mathrm{nbd})_{2}\right] \mathrm{SbF}_{6}$ $(5.2 \mathrm{mg}, 10 \mu \mathrm{mol})$, and $(S, S)-(R, R)-\operatorname{PhTRAP}(8.3 \mathrm{mg}, 10.5 \mu \mathrm{mol})$ to give 2c $(163 \mathrm{mg}, 93 \%)$. The enantiomeric excess of the product was determined to be $96 \%$ ee by HPLC analysis with a chiral stationary phase column, CHIRALCEL OD-H $(4.6 \mathrm{~mm} \phi \times 25 \mathrm{~cm}, 20 \%$ 2-propanol in hexane): colorless solid; $[\alpha]_{\mathrm{D}}^{25}=+88.9\left(c_{1} 1.01, \mathrm{CHCl}_{3}\right) ;{ }^{1} \mathrm{H} \mathrm{NMR}\left(400 \mathrm{MHz}, \mathrm{CDCl}_{3}, \mathrm{TMS}\right) \delta 2.39(\mathrm{~s}, 3 \mathrm{H})$, 3.74-3.82 (m, 1H), 4,31-4.38 (m, 2H), 6.84-6.89 (m, 3H), 6.97 (t, J=7.5 Hz, 1H), 7.17-7.28 (m, 6H), $7.68(\mathrm{~d}, J=8.0 \mathrm{~Hz}, 2 \mathrm{H}), 7.74(\mathrm{~d}, J=8.0 \mathrm{~Hz}, 1 \mathrm{H}) ;{ }^{13} \mathrm{C}\left\{{ }^{1} \mathrm{H}\right\} \mathrm{NMR}\left(100.5 \mathrm{MHz}, \mathrm{CDCl}_{3}\right) \delta 21.6,46.2$, 58.5, 114.9, 124.0, 125.6, 127.1, 127.4, 127.7, 128.3, 128.7, 129.7, 133.8, 134.8, 142.1, 142.4, 144.1; Anal. Calcd for $\mathrm{C}_{21} \mathrm{H}_{19} \mathrm{NO}_{2} \mathrm{~S}$ : C, 72.18; H, 5.48; N, 4.01. Found: C, 71.96; H, 5.46; N, 3.90 .

(+)-3-[2-(tert-butyldimethylsilyloxy)ethyl]-1-(p-toluenesulfonyl)indoline (2h) (Table 2, Entry 3 ). The general procedure was followed with 3-[2-(tert-butyldimethylsilyloxy)ethyl]-1-( $p$ toluenesulfonyl)indole (1d) $(215 \mathrm{mg}, 0.50 \mathrm{mmol})$ to give $\mathbf{2 d}(202 \mathrm{mg}, 94 \%)$. The enantiomeric excess of the product was determined to be $98 \%$ ee by HPLC analysis with a chiral stationary phase column, Ceramospher RU-1 (4.6 mm $\phi \times 25 \mathrm{~cm}$, methanol): colorless solid; $[\alpha]^{20}{ }_{\mathrm{D}}=+9.70\left(c 1.18, \mathrm{CHCl}_{3}\right) ;{ }^{1} \mathrm{H}$ NMR (300 MHz, $\left.\mathrm{CDCl}_{3}, \mathrm{TMS}\right) \delta 0.05(\mathrm{~s}, 6 \mathrm{H}), 0.90(\mathrm{~s}, 9 \mathrm{H}), 1.32-1.45(\mathrm{~m}, 1 \mathrm{H}), 1.70-1.82(\mathrm{~m}, 1 \mathrm{H})$, $2.32(\mathrm{~s}, 3 \mathrm{H}), 3.14-3.27(\mathrm{~m}, 1 \mathrm{H}), 3.57(\mathrm{dd}, J=6.6,10.8 \mathrm{~Hz}, 1 \mathrm{H}), 3.58(\mathrm{t}, J=6.0 \mathrm{~Hz}, 2 \mathrm{H}), 3.99(\mathrm{dd}, J=$ 9.0, 10.8, 1H), 6.94 (dt, $J=0.8,7.4 \mathrm{~Hz}, 1 \mathrm{H}), 7.02$ (d, $J=7.4 \mathrm{~Hz}, 1 \mathrm{H}), 7.12-7.21(\mathrm{~m}, 3 \mathrm{H}), 7.58-7.66$ $(\mathrm{m}, 3 \mathrm{H}) ;{ }^{13} \mathrm{C}\left\{{ }^{1} \mathrm{H}\right\} \mathrm{NMR}\left(75 \mathrm{MHz}, \mathrm{CDCl}_{3}\right) \delta-5.5,18.1,21.4,25.8,37.2,37.5,56.0,60.7,114.9,123.7$, 124.4, 127.4, 128.0, 129.7, 134.1, 135.7, 141.8, 144.0; Anal. Calcd for $\mathrm{C}_{23} \mathrm{H}_{33} \mathrm{NO}_{3} \mathrm{SSi}: \mathrm{C}, 64.00 ; \mathrm{H}$, 7.71; N, 3.24. Found: C, 64.27; H, 7.86; N, 3.33.

(+)-3-[2-(tert-butoxycarbonyl)ethyl]-1-(p-toluenesulfonyl)indoline (2i) (Table 2, Entry 4). The general procedure was followed with 3-[2-(tert-butoxycarbonyl)ethyl]-1-( $p$-toluenesulfonyl)indole (1e) $(201 \mathrm{mg}, 0.50 \mathrm{mmol})$ to give $2 \mathbf{e}(187 \mathrm{mg}, 93 \%)$. The enantiomeric excess of the product was determined to be $97 \%$ ee by HPLC analysis with a chiral stationary phase column, CHIRALPAK AD (4.6 mm $\phi \times 25 \mathrm{~cm}, 4 \%$ 2-propanol in hexane): colorless solid; $[\alpha]^{20}{ }_{\mathrm{D}}=+15.6\left(c 1.06, \mathrm{CHCl}_{3}\right) ;{ }^{1} \mathrm{H}$ NMR (300 MHz, CDCl 3 , TMS) $\delta 1.43(\mathrm{~s}, 9 \mathrm{H}), 1.47-1.60(\mathrm{~m}, 1 \mathrm{H}), 1.79-1.92(\mathrm{~m}, 1 \mathrm{H}), 2.16(\mathrm{t}, J=7.7$ $\mathrm{Hz}, 2 \mathrm{H}), 2.36(\mathrm{~s}, 3 \mathrm{H}), 3.08-3.20(\mathrm{~m}, 1 \mathrm{H}), 3.58(\mathrm{dd}, J=5.9,10.4 \mathrm{~Hz}, 1 \mathrm{H}), 3.97$ (dd, $J=9.0,10.4 \mathrm{~Hz}$, $1 \mathrm{H}), 6.99(\mathrm{dt}, J=0.9,7.5 \mathrm{~Hz}, 1 \mathrm{H}), 7.09(\mathrm{~d}, J=7.5 \mathrm{~Hz}, 1 \mathrm{H}), 7.18-7.26(\mathrm{~m}, 3 \mathrm{H}), 7.65(\mathrm{~d}, J=8.4 \mathrm{~Hz}$, $1 \mathrm{H}), 7.69(\mathrm{~d}, J=8.1 \mathrm{~Hz}, 2 \mathrm{H}) ;{ }^{13} \mathrm{C}\left\{{ }^{1} \mathrm{H}\right\} \mathrm{NMR}\left(75 \mathrm{MHz}, \mathrm{CDCl}_{3}\right) \delta 21.4,28.0,29.7,32.5,39.0,55.2$, 80.6, 114.8, 123.7, 124.6, 127.3, 128.2, 129.7, 133.8, 134.7, 141.7, 144.2, 172.2; Anal. Calcd for $\mathrm{C}_{22} \mathrm{H}_{27} \mathrm{NO}_{4} \mathrm{~S}: \mathrm{C}, 65.81 ; \mathrm{H}, 6.78 ; \mathrm{N}, 3.49$. Found: $\mathrm{C}, 66.03 ; \mathrm{H}, 6.82 ; \mathrm{N}, 3.50$.

(+)-3-[2-\{ $N$-(tert-butoxycarbonyl)amino\}ethyl]-1-(p-toluenesulfonyl)indoline (2j) (Table 2 , Entry 5). The general procedure was followed with 3-[2- $\{N$-(tert-butoxycarbonyl)amino $\}$ ethyl $]-1-(p-$ toluenesulfonyl)indole (1f) (208 $\mathrm{mg}, 0.50 \mathrm{mmol})$ to give $\mathbf{2 e}(148 \mathrm{mg}, 71 \%)$. The enantiomeric excess of the product was determined to be $95 \%$ ee by HPLC analysis with a chiral stationary phase column, CHIRALCEL OC $\left(4.6 \mathrm{~mm} \phi \times 25 \mathrm{~cm}, 20 \%\right.$ 2-propanol in hexane): colorless solid; $[\alpha]_{\mathrm{D}}^{20}=+9.0(c$ 1.02, $\left.\mathrm{CHCl}_{3}\right) ;{ }^{1} \mathrm{H}$ NMR $\left(300 \mathrm{MHz}, \mathrm{CDCl}_{3}\right.$, TMS) $\delta 1.32-1.55(\mathrm{~m}, 1 \mathrm{H}), 1.44(\mathrm{~s}, 9 \mathrm{H}), 1.67-1.80(\mathrm{~m}, 1 \mathrm{H})$, $2.37(\mathrm{~s}, 3 \mathrm{H}), 3.05-3.20(\mathrm{~m}, 3 \mathrm{H}), 3.61(\mathrm{dd}, J=5.9,10.6 \mathrm{~Hz}, 1 \mathrm{H}), 4.00$ (dd, $J=9.2,10.6,1 \mathrm{H}), 4.48$ (br s, $1 \mathrm{H}), 6.99(\mathrm{t}, J=7.4 \mathrm{~Hz}, 1 \mathrm{H}), 7.08(\mathrm{~d}, J=7.4 \mathrm{~Hz}, 1 \mathrm{H}), 7.17-7.26(\mathrm{~m}, 3 \mathrm{H}), 7.65(\mathrm{~d}, J=8.1 \mathrm{~Hz}, 1 \mathrm{H})$, 
$7.69(\mathrm{~d}, J=8.4 \mathrm{~Hz}, 2 \mathrm{H}) ;{ }^{13} \mathrm{C}\left\{{ }^{1} \mathrm{H}\right\} \mathrm{NMR}\left(75 \mathrm{MHz}, \mathrm{CDCl}_{3}\right) \delta 21.4,28.3,29.6,35.3,37.6,38.2$, 55.4, $114.9,123.8,124.4,127.4,128.2,129.7,133.9,134.9,141.7,144.2$, 155.9; Anal. Calcd for $\mathrm{C}_{22} \mathrm{H}_{28} \mathrm{~N}_{2} \mathrm{O}_{4} \mathrm{~S}$ : C, 63.44; H, 6.78; N, 6.73. Found: C, 63.71; H, 6.83; N, 6.60.

Removal of $N$-Tosyl Group from 2c. Sodium bis(2-methoxyethoxy)aluminum dihydride ( $c a$. $70 \%$ in toluene) $(1.10 \mathrm{~g}, 3.8 \mathrm{mmol})$ was added to a solution of $(R)-\mathbf{2 c}(273 \mathrm{mg}, 0.95 \mathrm{mmol})$ in toluene $(2.0 \mathrm{ml})$. The mixture was stirred under reflux for $14 \mathrm{~h}$. After the reaction was quenched with $1 \mathrm{~N} \mathrm{HCl}$ aq $(2 \mathrm{ml})$, the mixture was stirred for $1 \mathrm{~h}$ at room temperature. After filtration through Cerite, the filtrate was extracted three times with $1 \mathrm{~N} \mathrm{HCl}$ aq. The combined aqueous layer was basified with $\mathrm{Na}_{2} \mathrm{CO}_{3}$ and was extacted three times with EtOAc. The combined organic layer was dried with $\mathrm{Na}_{2} \mathrm{SO}_{4}$ and was evaporated, giving $(R)-3$-methylindoline $(82 \mathrm{mg}, 65 \%)$ as colorless oil: $[\alpha]_{\mathrm{D}}^{25}=-38.8(c 1.04$, $\left.\mathrm{CHCl}_{3}\right) ;{ }^{1} \mathrm{H} \mathrm{NMR}\left(400 \mathrm{MHz}, \mathrm{CDCl}_{3}, \mathrm{TMS}\right) \delta 1.32(\mathrm{~d}, J=6.9 \mathrm{~Hz}, 3 \mathrm{H}), 3.10(\mathrm{t}, J=8.6 \mathrm{~Hz}, 1 \mathrm{H}), 3.2-4.0$ (br s, 1H), 3.36 (sextet, $J=7.5 \mathrm{~Hz}, 1 \mathrm{H}), 3.69(\mathrm{t}, J=8.7 \mathrm{~Hz}, 1 \mathrm{H}), 6.64(\mathrm{~d}, \mathrm{~J}=7.7 \mathrm{~Hz}, 1 \mathrm{H}), 6.73(\mathrm{t}, J=$ $7.7 \mathrm{~Hz}, 1 \mathrm{H}), 7.02(\mathrm{t}, J=7.6,1 \mathrm{H}), 7.08(\mathrm{~d}, J=7.3 \mathrm{~Hz}, 1 \mathrm{H}) ;{ }^{13} \mathrm{C}\left\{{ }^{1} \mathrm{H}\right\} \mathrm{NMR}\left(100.5 \mathrm{MHz}, \mathrm{CDCl}_{3}\right) \delta 18.6$, $36.6,55.4,109.5,118.7,123.3,127.2,134.3,151.2$. The enantiomeric excess of the indoline was determined to be $98 \%$ ee by HPLC analysis of its acetamide with a chiral stationary phase column, CHIRALCEL OC (4.6 mm $\phi \times 25 \mathrm{~cm}, 20 \%$ 2-propanol in hexane).

$\boldsymbol{N}$-Methanesulfonyl-2-iodo-5-methoxyaniline (6). ${ }^{5}$ Methanesulfonylchloride (3.78 g, $\left.33 \mathrm{mmol}\right)$ was added dropwise for $10 \mathrm{~min}$ to a mixture of 2-iodo-5-methoxyaniline (5) $(7.47 \mathrm{~g}, 30 \mathrm{mmol})$ and pyridine $(2.61 \mathrm{~g}, 33 \mathrm{mmol})$ in $\mathrm{CH}_{2} \mathrm{Cl}_{2}$ at $0{ }^{\circ} \mathrm{C}$, and the mixture was stirred at room temperature for $16 \mathrm{~h}$. After water was added, the mixture was extracted with EtOAc. The organic layer was washed with brine, was dried with $\mathrm{Na}_{2} \mathrm{SO}_{4}$, and was evaporated. The residue was purified by a flash column chromatography on silica gel (hexanes/EtOAc), giving the desired product $6(8.13 \mathrm{~g}, 83 \%)$ as a pale brown solid: ${ }^{1} \mathrm{H} \mathrm{NMR}\left(400 \mathrm{MHz}, \mathrm{CDCl}_{3}, \mathrm{TMS}\right) \delta 3.01(\mathrm{~s}, 3 \mathrm{H}), 3.81(\mathrm{~s}, 3 \mathrm{H}), 6.56(\mathrm{dd}, J=3.0,8.6 \mathrm{~Hz}$, $1 \mathrm{H}), 6.52(\mathrm{br} \mathrm{s}, 1 \mathrm{H}), 7.26(\mathrm{~d}, J=3.0 \mathrm{~Hz}, 1 \mathrm{H}), 7.66(\mathrm{~d}, J=8.6 \mathrm{~Hz}, 1 \mathrm{H}) ;{ }^{13} \mathrm{C}\left\{{ }^{1} \mathrm{H}\right\} \mathrm{NMR}(100.5 \mathrm{MHz}$, $\left.\mathrm{CDCl}_{3}\right) \delta 39.9,55.5,80.0,107.9,113.8,138.3,139.3,161.0$.

N-Methanesulfonyl-6-methoxy-3-[(triisopropylsilyloxy)methyl]indole (8). Trimethyl[3(triisopropylsilyloxy)-1-propynyl]silane (7) $(4.27 \mathrm{~g}, 15 \mathrm{mmol})$ was added to a mixture of 6 (1.64 g, 5.0 $\mathrm{mmol}), \mathrm{NaHCO}_{3}(2.10 \mathrm{~g}, 25 \mathrm{mmol}), \mathrm{LiCl}(212 \mathrm{mg}, 5.0 \mathrm{mmol})$, and palladium acetate $(56 \mathrm{mg}, 0.25$ $\mathrm{mmol})$ in DMF $(100 \mathrm{ml})$. After $4.5 \mathrm{~h}$ of stirring at $100{ }^{\circ} \mathrm{C}$, the mixture was diluted with EtOAc and saturated $\mathrm{NH}_{4} \mathrm{Cl}$ aq., and then the aqueous layer was saturated with $\mathrm{NaCl}$. After the mixture was filtered through Celite, the filtrate was extracted three times with EtOAc. The combined organic layer was dried with $\mathrm{Na}_{2} \mathrm{SO}_{4}$ and was evaporated. A mixture of the black oily residue, potassium acetate $(2.45 \mathrm{~g}, 25 \mathrm{mmol})$, and tetrabutylammonium chloride $(1.39 \mathrm{~g}, 5.0 \mathrm{mmol})$ in DMF $(90 \mathrm{ml})$ was stirred at $100{ }^{\circ} \mathrm{C}$ for $4 \mathrm{~h}$. The mixture was diluted with EtOAc and saturated $\mathrm{NH}_{4} \mathrm{Cl}$ aq., and then the aqueous layer was saturated with $\mathrm{NaCl}$. After the mixture was filtered through Celite, the filtrate was extracted three times with EtOAc. The combined organic layer was dried with $\mathrm{Na}_{2} \mathrm{SO}_{4}$ and was evaporated. The residue was purified by a flash column chromatography on silica gel (hexanes/EtOAc), giving the desired product $6(1.25 \mathrm{~g}, 61 \%)$ as brown oil: ${ }^{1} \mathrm{H}$ NMR $\left(400 \mathrm{MHz}, \mathrm{CDCl}_{3}, \mathrm{TMS}\right) \delta 1.09(\mathrm{~d}, J=6.6 \mathrm{~Hz}$, $18 \mathrm{H}), 1.13-1.24(\mathrm{~m}, 3 \mathrm{H}), 3.04(\mathrm{~s}, 3 \mathrm{H}), 3.88(\mathrm{~s}, 3 \mathrm{H}), 4.93(\mathrm{~s}, 2 \mathrm{H}), 6.94(\mathrm{dd}, J=2.3,8.7 \mathrm{~Hz}, 1 \mathrm{H}), 7.25$ $(\mathrm{s}, 1 \mathrm{H}), 7.44(\mathrm{~d}, J=2.3 \mathrm{~Hz}, 1 \mathrm{H}), 7.51(\mathrm{~d}, J=8.7 \mathrm{~Hz}, 1 \mathrm{H}) ;{ }^{13} \mathrm{C}\left\{{ }^{1} \mathrm{H}\right\} \mathrm{NMR}\left(100.5 \mathrm{MHz}, \mathrm{CDCl}_{3}\right) \delta 12.0$, 18.0, 40.1, 55.8, 58.4, 97.4, 112.6, 120.8, 121.3, 123.2, 123.5, 136.6, 158.3; Anal. Calcd for $\mathrm{C}_{20} \mathrm{H}_{33} \mathrm{NO}_{4} \mathrm{SSi}: \mathrm{C}, 58.36 ; \mathrm{H}, 8.08 ; \mathrm{N}, 3.40$. Found: C, 58.66; H, 8.20; N, 3.29.

(+)-N-Methanesulfonyl-6-methoxy-3-[triisopropylsilyloxymethyl]indoline (9). The general procedure of the asymmetric hydrogenation of was followed with $\mathbf{8}$ (412 $\mathrm{mg}, 1.00 \mathrm{mmol})$ to give the desired product $9(355 \mathrm{mg}, 87 \%)$. The enantiomeric excess of the product was determined to be $93 \%$ ee by HPLC analysis with a chiral stationary phase column, CHIRALCEL OD-H $(4.6 \mathrm{~mm} \phi \times 25 \mathrm{~cm}$, 
$10 \%$ 2-propanol in hexane): colorless oil; $[\alpha]_{\mathrm{D}}^{25}=+27.0\left(\right.$ c $\left.1.05, \mathrm{CHCl}_{3}\right) ;{ }^{1} \mathrm{H} \mathrm{NMR}\left(400 \mathrm{MHz}, \mathrm{CDCl}_{3}\right.$, TMS) $\delta$ 0.99-1.14 (m, 21H), $2.88(\mathrm{~s}, 3 \mathrm{H}), 3.44-3.53(\mathrm{~m}, 1 \mathrm{H}), 3.73(\mathrm{dd}, J=7.6,9.6 \mathrm{~Hz}, 1 \mathrm{H}), 3.80(\mathrm{~s}$, $3 \mathrm{H}), 3.84(\mathrm{dd}, J=5.8,9.6 \mathrm{~Hz}, 1 \mathrm{H}), 3.94(\mathrm{dd}, J=5.1,10.7 \mathrm{~Hz}, 1 \mathrm{H}), 4.02(\mathrm{dd}, J=9.1,10.7 \mathrm{~Hz}, 1 \mathrm{H})$, $6.57(\mathrm{dd}, J=2.4,8.5 \mathrm{~Hz}, 1 \mathrm{H}), 7.02(\mathrm{~d}, J=2.4 \mathrm{~Hz}, 1 \mathrm{H}), 7.12(\mathrm{~d}, J=8.5 \mathrm{~Hz}, 1 \mathrm{H}) ;{ }^{13} \mathrm{C}\left\{{ }^{1} \mathrm{H}\right\} \mathrm{NMR}(100.5$ $\left.\mathrm{MHz}, \mathrm{CDCl}_{3}\right) \delta 11.9,17.9,34.3,42.4,54.0,55.6,65.7,100.0,109.0,123.8,125.6,143.4$, 160.5; Anal. Calcd for $\mathrm{C}_{20} \mathrm{H}_{35} \mathrm{NO}_{4} \mathrm{SSi}$ : C, 58.07; H, 8.53; N, 3.39. Found: C, 57.87; H, 8.54; N, 3.32.

(+)- $\mathrm{N}$-Methanesulfonyl-6-methoxy-3-(hydroxymethyl)indoline. A solution of 9 (549 mg, 1.33 $\mathrm{mmol})$ in THF $(2 \mathrm{ml}), \mathrm{H}_{2} \mathrm{O}(2 \mathrm{ml})$, and trifluoroacetic acid $(0.67 \mathrm{ml})$ was stirred at $50{ }^{\circ} \mathrm{C}$ for $5 \mathrm{~h}$. The mixture was diluted with brine, and was extracted three times with EtOAc. The combined organic layer was dried with $\mathrm{Na}_{2} \mathrm{SO}_{4}$, and was evaporated. The residue was purified by a flash column chromatography on silica gel (hexanes/EtOAc), giving the desired product (274 $\mathrm{mg}, 80 \%$ ) as pale blue oil: $[\alpha]_{D}^{25}=+22.4\left(c 0.90, \mathrm{CHCl}_{3}\right) ;{ }^{1} \mathrm{H} \mathrm{NMR}\left(400 \mathrm{MHz}, \mathrm{CDCl}_{3}, \mathrm{TMS}\right) \delta 1.98$ (br s, 1H), 2.90 (s, 3H), $3.44-3.52(\mathrm{~m}, 1 \mathrm{H}), 3.75(\mathrm{dd}, J=6.0,11.2 \mathrm{~Hz}, 1 \mathrm{H}), 3.77(\mathrm{dd}, J=5.6,11.2 \mathrm{~Hz}, 1 \mathrm{H}), 3.79(\mathrm{~s}, 3 \mathrm{H}), 3.95$ $(\mathrm{dd}, J=5.0,10.9 \mathrm{~Hz}, 1 \mathrm{H}), 4.06(\mathrm{dd}, J=9.1,10.9 \mathrm{~Hz}, 1 \mathrm{H}), 6.59(\mathrm{dd}, J=2.4,8.3 \mathrm{~Hz}, 1 \mathrm{H}), 7.02(\mathrm{~d}, J=$ $2.4 \mathrm{~Hz}, 1 \mathrm{H}), 7.13(\mathrm{~d}, J=8.3 \mathrm{~Hz}, 1 \mathrm{H}) ;{ }^{13} \mathrm{C}\left\{{ }^{1} \mathrm{H}\right\} \mathrm{NMR}\left(100.5 \mathrm{MHz}, \mathrm{CDCl}_{3}\right) \delta 34.5,41.7,53.8,55.6$, 64.6, 100.2, 109.2, 123.1, 125.4, 143.6, 160.6; Anal. Calcd for $\mathrm{C}_{11} \mathrm{H}_{15} \mathrm{NO}_{4} \mathrm{~S}: \mathrm{C}, 51.35 ; \mathrm{H}, 5.88 ; \mathrm{N}, 5.44$. Found: C, 51.31; H, 5.88; N, 5.46.

(+)- $N$-Methanesulfonyl-6-methoxy-3-(acetoxymethyl)indoline (4). Acetic anhydride (84 mg, $0.82 \mathrm{mmol})$ and triethylamine $(90 \mathrm{mg}, 0.89 \mathrm{mmol})$ was added to a mixture of (+)- $N$-methanesulfonyl-6methoxy-3-(hydroxymethyl)indoline $(191 \mathrm{mg}, 0.74 \mathrm{mmol}$ ) and DMAP (4.5 mg, $37 \mu \mathrm{mol})$ in $\mathrm{CH}_{2} \mathrm{Cl}_{2}$ $(0.4 \mathrm{ml})$. After $3 \mathrm{~h}$ of stirring at room temperature, the mixture was diluted with $1 \mathrm{~N} \mathrm{HCl} \mathrm{aq.} \mathrm{and} \mathrm{was}$ extracted three times with EtOAc. The combined organic layer was washed with brine, was dried with $\mathrm{Na}_{2} \mathrm{SO}_{4}$, and was evaporated. The residue was purified by a flash column chromatography on silica gel (hexanes/EtOAc), giving the desired product $(208 \mathrm{mg}, 94 \%)$ as pale yellow oil: $[\alpha]_{\mathrm{D}}^{25}=+30.0(c 1.09$, $\left.\mathrm{CHCl}_{3}\right) ;{ }^{1} \mathrm{H}$ NMR (400 MHz, CDCl 3 , TMS) $\delta 2.08(\mathrm{~s}, 3 \mathrm{H}), 2.91(\mathrm{~s}, 3 \mathrm{H}), 3.59-3.67(\mathrm{~m}, 1 \mathrm{H}), 3.80$ (s, $3 \mathrm{H}), 3.87(\mathrm{dd}, J=5.3,10.7 \mathrm{~Hz}, 1 \mathrm{H}), 4.03-4.12(\mathrm{~m}, 2 \mathrm{H}), 4.26(\mathrm{dd}, J=5.4,11.1 \mathrm{~Hz}, 1 \mathrm{H}), 6.59(\mathrm{dd}, J=$ $2.6,8.6 \mathrm{~Hz}, 1 \mathrm{H}), 7.03(\mathrm{~d}, J=2.6 \mathrm{~Hz}, 1 \mathrm{H}), 7.12(\mathrm{~d}, J=8.6 \mathrm{~Hz}, 1 \mathrm{H}) ;{ }^{13} \mathrm{C}\left\{{ }^{1} \mathrm{H}\right\} \mathrm{NMR}(100.5 \mathrm{MHz}$, $\left.\mathrm{CDCl}_{3}\right) \delta 20.8,34.6,38.9,54.2,55.6,65.9,100.2$, 109.4, 122.2, 125.6, 143.3, 160.9, 170.8; Anal. Calcd for $\mathrm{C}_{13} \mathrm{H}_{17} \mathrm{NO}_{5} \mathrm{~S}$ : C, 52.16; H, 5.72; N, 4.68. Found: C, 51.93; H, 5.75; N, 4.69.

Assignment of Absolute Configuration of 2c. The absolute configuration of 2c obtained from the asymmetric hydrogenation of 1c using $(S, S)-(R, R)$-PhTRAP was assigned to be $S$ as follows: (1) 2c was converted into $N$-benzyl-3-methylindoline. (2) The specific rotation of the sample derived from 2c was compared to the value of specific rotation of $(R)-2 \mathrm{c}$ in the literature. ${ }^{6}$

$N$-Benzyl-3-methylindoline. Sodium bis(2-methoxyethoxy)aluminum dihydride $(\mathrm{ca}$. $70 \%$ in toluene) (324 g, $1.12 \mathrm{mmol})$ was added to a solution of $(R)-2 \mathrm{c}(72 \mathrm{mg}, 0.25 \mathrm{mmol})$ in toluene $(0.5 \mathrm{ml})$. The mixture was stirred under reflux for $5 \mathrm{~h}$. After dilution with $\mathrm{H}_{2} \mathrm{O}$ and EtOAc, the mixture was filtered through Cerite. The filtrate was extracted three times with EtOAc. The combined organic layer was dried with $\mathrm{Na}_{2} \mathrm{SO}_{4}$ and was evaporated. Benzyl chloride (48 $\mathrm{mg}, 0.38 \mathrm{mmol}$ ) was added to a solution of the residue, $\mathrm{K}_{2} \mathrm{CO}_{3}(57 \mathrm{mg}, 0.41 \mathrm{mmol})$, and $\mathrm{KI}(4.5 \mathrm{mg}, 27 \mu \mathrm{mol})$ in DMF $(0.5 \mathrm{ml})$. After $5 \mathrm{~h}$ of stirring at $100{ }^{\circ} \mathrm{C}$, the mixture was diluted with $\mathrm{H}_{2} \mathrm{O}$, and was extracted ten times with hexanes. The combined organic layer was washed with $\mathrm{H}_{2} \mathrm{O}$, was dried with $\mathrm{Na}_{2} \mathrm{SO}_{4}$, and was evaporated. After passing through a column on silica gel, the residue was purified by medium-pressure liquid chromatography (hexanes/EtOAc) with C.I.G. pre-packed column CPS-223L-1 (Kusano, Tokyo, Japan), giving the desired compounds $(21 \mathrm{mg}, 38 \%)$ as pale yellow oil: $[\alpha]_{\mathrm{D}}^{25}=+66.4(c 0.51$, $\left.\mathrm{CH}_{2} \mathrm{Cl}_{2}\right)$, lit. ${ }^{6}[\alpha]_{\mathrm{D}}^{20}=-49.7\left(c 1, \mathrm{CH}_{2} \mathrm{Cl}_{2}\right)$ for $87 \%$ ee $(R) ;{ }^{1} \mathrm{H} \mathrm{NMR}\left(400 \mathrm{MHz}, \mathrm{CDCl}_{3}\right.$, TMS) $\delta 1.30(\mathrm{~d}$, $J=6.5 \mathrm{~Hz}, 3 \mathrm{H}), 2.83(\mathrm{t}, J=8.5 \mathrm{~Hz}, 1 \mathrm{H}), 3.30$ (sextet, $J=7.5 \mathrm{~Hz}, 1 \mathrm{H}), 3.50(\mathrm{t}, J=8.5 \mathrm{~Hz}, 1 \mathrm{H}), 4.11(\mathrm{~d}$, $J=15.1 \mathrm{~Hz}, 1 \mathrm{H}), 4.36(\mathrm{~d}, J=15.1 \mathrm{~Hz}, 1 \mathrm{H}), 6.51(\mathrm{~d}, J=8.0 \mathrm{~Hz}, 1 \mathrm{H}), 6.70(\mathrm{t}, J=7.5 \mathrm{~Hz}, 1 \mathrm{H}), 7.06(\mathrm{t}, J$ 
$=7.0 \mathrm{~Hz}, 2 \mathrm{H}), 7.24-7.36(\mathrm{~m}, 5 \mathrm{H}) ;{ }^{13} \mathrm{C}\left\{{ }^{1} \mathrm{H}\right\} \mathrm{NMR}\left(100.5 \mathrm{MHz}, \mathrm{CDCl}_{3}\right) \delta$ 18.6, 35.2, 53.4, 61.6, 107.0, $117.7,123.2,127.0,127.4,127.9,128.4,135.0,138.5,152.1$.

(1) Schrock, R. R.; Osborn, J. A. J. Am. Chem. Soc. 1971, 93, 3089-3091.

(2) Sawamura, M.; Hamashima, H.; Sugawara, M.; Kuwano, R.; Ito, Y. Organometallics 1995, 14, 4549-4558.

(3) Ma, C.; Liu, X.; Li, X.; Flippen-Anderson, J.; Yu, S.; Cook, J. M. J. Org. Chem. 2001, 66, 4525-4542.

(4) Jones, T. K.; Denmark, S. E. Org. Synth. 1986, 64, 182-188.

(5) Kizil, M.; Patro, B.; Callaghan, O.; Murphy, J. A.; Hursthouse, M. B.; Hibbs, D. J. Org. Chem. 1999, 64, 7856-7862.

(6) Gil, G. S.; Groth, U. M. J. Am. Chem. Soc. 2000, 122, 6789-6790. 